\title{
ARTICLE OPEN Rethinking phonons: The issue of disorder
}

Hamid Reza Seyf ${ }^{1}$, Luke Yates ${ }^{1}$, Thomas L. Bougher ${ }^{2}$, Samuel Graham ${ }^{1,2,3}$, Baratunde A. Cola ${ }^{1,2,3}$, Theeradetch Detchprohm ${ }^{4}$, Mi-Hee Ji $^{4}$, Jeomoh Kim ${ }^{4}$, Russell Dupuis ${ }^{4}$, Wei Lv ${ }^{1}$ and Asegun Henry ${ }^{1,2,3}$

Current understanding of phonons treats them as plane waves/quasi-particles of atomic vibration that propagate and scatter. The problem is that conceptually, when any level of disorder is introduced, whether compositional or structural, the character of vibrational modes in solids changes, yet nearly all theoretical treatments continue to assume phonons are still waves. For example, the phonon contributions to alloy thermal conductivity (TC) rely on this assumption and are most often computed from the virtual crystal approximation (VCA). Good agreement is obtained in some cases, but there are many instances where it fails-both quantitatively and qualitatively. Here, we show that the conventional theory and understanding of phonons requires revision, because the critical assumption that all phonons/normal modes resemble plane waves with well-defined velocities is no longer valid when disorder is introduced. Here we show, surprisingly, that the character of phonons changes dramatically within the first few percent of impurity concentration, beyond which phonons more closely resemble the modes found in amorphous materials. We then utilize a different theory that can treat modes with any character and experimentally confirm its new insights.

npj Computational Materials (2017)3:49; doi:10.1038/s41524-017-0052-9

\section{INTRODUCTION}

The dominant mechanism for heat conduction in non-metals is via atomic vibrations. In solids, the vibrations experienced by an atom are a superposition of collective motions that groups of atoms make together at a single frequency, which are often termed normal modes of vibration. ${ }^{1-3}$ The amplitudes of these normal modes follow the rules of quantum mechanics, occurring in integer steps with different energy levels. ${ }^{1-3}$ Each step in vibrational mode energy is then thought of as a quasi-particle termed a phonon, and the theory describing their transport is known as the phonon gas model (PGM). ${ }^{1-9}$ The PGM was largely born out of the types of vibrations that would exist in an infinitely large, pure, homogeneous crystal (IPHC). In such a system, one can solve the equations of motion in the harmonic limit and find that all solutions correspond to plane wave modulated vibrations, as a result of the periodicity. If one adds together solutions with similar wave vectors, one then obtains a wave packet that propagates energy at the group velocity $\left(\frac{d \omega}{d \mathbf{k}}\right)$ and resembles a particle moving. The essence of the PGM is then that it assumes the energy of the vibrational modes can be treated as analogous to particles that travel and scatter with each other, similar to a gas of molecules-hence the name "phonon gas model".

According to the PGM the heat flux carried by a phonon is described by the product of its energy, its group velocity, divided by the volume it occupies, $\mathbf{Q}=\frac{1}{V}(h \omega) \mathbf{v}$, and this is the underlying assumption for virtually all theoretical expressions of phonon transport. $^{1-3,5,8,10}$ The validity of this assumption has been in many ways validated ${ }^{5,11-20}$ and consequently, the PGM has been used almost ubiquitously to understand phonon transport in all classes of solids. ${ }^{13,18,19,21-25}$ However, here we will examine more deeply the behaviors in a random alloy as a representative example, because its compositional disorder reveals a rather fundamental issue with the way phonons have been conceptualized, namely considering them to be plane waves/quasi-particles that travel and scatter. Our revision to this fundamental issue with the common PGM-based theory/conception of phonons is then tested by comparing to experiments. Our alternative perspective is based on correlation rather than scattering and yields excellent agreement with the experimental data. The inaccuracy of the scattering-based perspective vs. the consistent accuracy of the correlation-based perspective strongly suggests that a rethinking of how phonons behave in general is warranted, and a correlationbased physical picture is likely to be more useful and insightful.

The current theory: the virtual crystal approximation (VCA)

The current theory for phonon transport in alloys is based on the VCA. In the VCA, one essentially treats the alloyed system, which is compositionally a mixture of two or more pure crystals, as an effective crystal that has phonon properties (i.e., dispersion, velocities, specific heat etc.) that are compositionally weighted averages of the phonon properties of the constituent base crystals. However, the thermal conductivity (TC) of crystalline alloys is not well described by a simple rule of mixtures of the base crystal TCs. Instead, the VCA accounts for the deviation from such a simplified model by superimposing an additional mechanism for phonon scattering, namely that of compositional impurities/ defects. In this sense, the VCA treats the presence of dissimilar elements in an alloy lattice as though they act as scattering centers for the phonon gas, and the expressions used to model this effect were derived by Tamura ${ }^{26}$ in the context of modeling isotope scattering. This additional scattering mechanism then ultimately yields much more frequent scattering, which reduces the TC in much better agreement with experimental data, ${ }^{13,22,23,25}$ as compared to a simple rule of mixtures. The impurity scattering

\footnotetext{
${ }^{1}$ George W. Woodruff School of Mechanical Engineering, Georgia Institute of Technology, Atlanta, GA, USA; ${ }^{2}$ Heat Lab, Georgia Institute of Technology, Atlanta, GA, USA; ${ }^{3}$ School

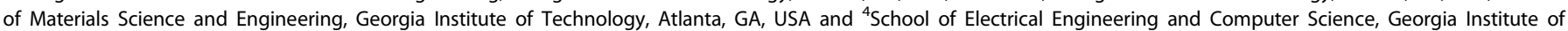
Technology, Atlanta, GA, USA

Correspondence: Asegun Henry (ase@gatech.edu)
}

Received: 27 April 2017 Revised: 7 October 2017 Accepted: 22 October 2017

Published online: 16 November 2017 

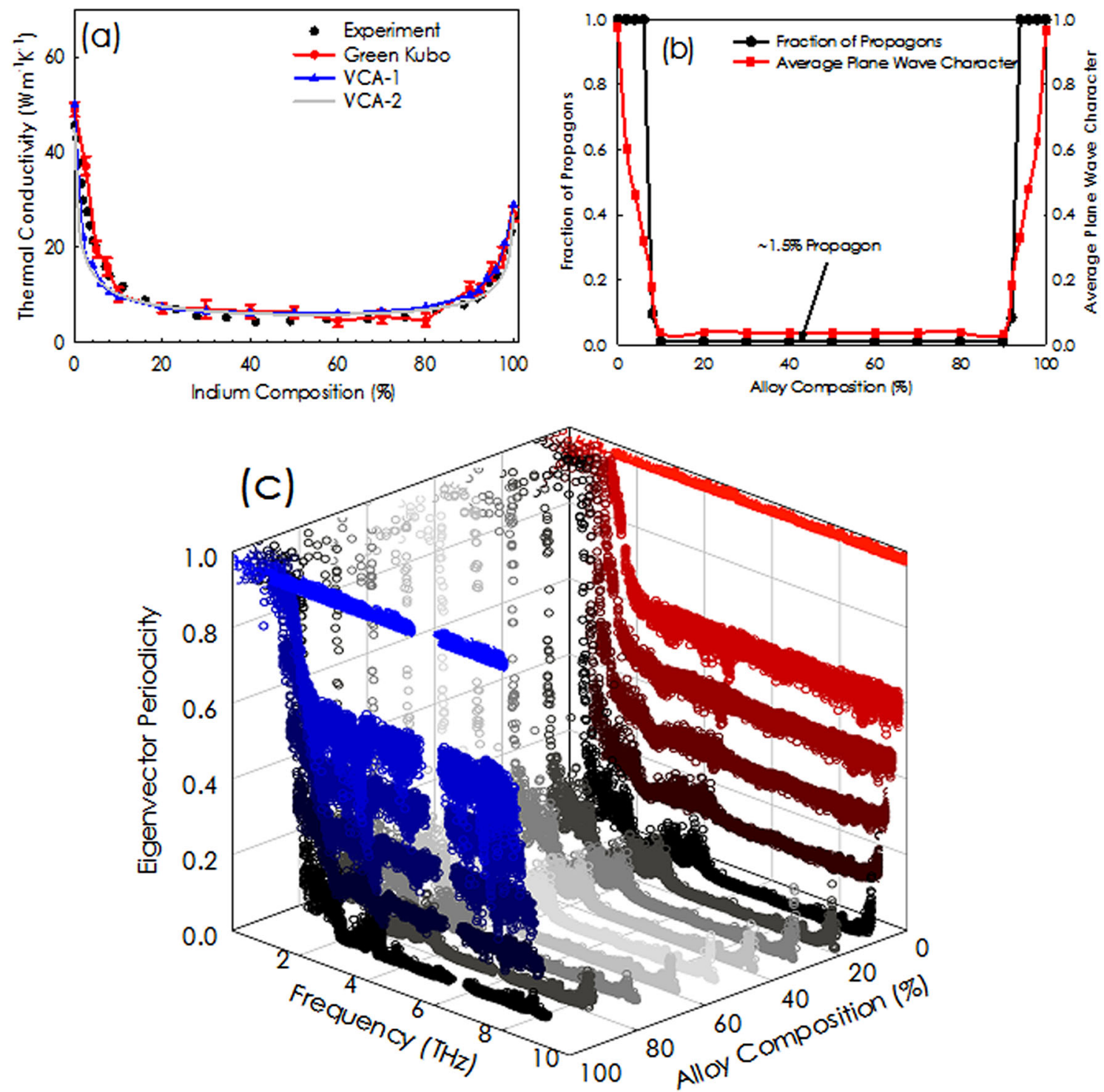

Fig. 1 Thermal Conductivity (TC) and the eigenvector periodicity parameter for $\ln _{x} G a_{1-x}$ As. a TC of $\ln _{x} G a_{1-x} A s$ vs. Indium composition at $300 \mathrm{~K}$ as predicted by the VCA using the Tersoff empirical potential, VCA using fitting parameters, molecular dynamics simulations using the Tersoff potential and the Green Kubo (GK) formalism, as compared to experiments. ${ }^{55}$ The GK error bars were determined based on the standard deviation of results from independent simulations at a given Indium composition. Here, it should be noted that at $300 \mathrm{~K}$ there is a minimal effect from quantum corrections and thus, to reduce computational expense, GK as opposed to GKMA calculations were performed, b Average eigenvector periodicity parameter and fraction of propagating modes vs. Indium composition, c Eigenvector periodicity parameter for different alloy compositions

term is then what qualitatively causes the VCA to correctly predict the typical U-shaped curve observed for TC vs. composition in most alloys (see Fig. 1a). ${ }^{13,22,23,25}$ For alloys, TC typically decreases by $\sim 10$ times as the composition of a single impurity approaches $\sim 10-25 \%$ and then it remains approximately constant until approximately $75-90 \%$ after which it quickly increases to the other pure crystal's TC (see Fig. 1a).

Until the more recent pioneering work of Garg and Marzari, ${ }^{22}$ the approach of modeling alloy TC lacked predictive capability, because both the process of determining the phonon-phonon scattering rates and the defect scattering rates relied on fitting to existing experimental data. Thus, comparisons with experimental data often yielded good agreement, particularly when compared to the alloy TC vs. composition (see Fig. 1a). However, with the $a b$ initio approach developed by Broido and coworkers ${ }^{16,19,25}$ as well as Esfarjani and coworkers, ${ }^{14,19}$ one can now evaluate the two scattering mechanisms (e.g., phonon-phonon anharmonic scattering and phonon impurity scattering-often termed mass disorder scattering) without fitting to any experimental data. As a result, the VCA still represents the most advanced theoretical understanding of phonon transport in alloys, and this has largely been justified by the instances in the literature, where good agreement between the VCA and experiments has been observed. $^{22-25,27,28}$

Unfortunately there are instances where the VCA fails, ${ }^{29-32}$ even when adjustable parameters are used to fit the data to which it is compared. ${ }^{30,31}$ It is also important to note that these failures are not only quantitative, ${ }^{29-32}$ but also qualitative, ${ }^{29,30,32}$ particularly with respect to describing TC vs. temperature. This is because some alloys exhibit monotonically increasing TC or seemingly constant TC vs. temperature, ${ }^{29,30,32}$ and the VCA will rather inherently always yield the same qualitative temperature dependence as a pure crystal, which consists of a low-temperature peak (dictated by the competition between phonon-phonon scattering and phonon-impurity scattering) followed by monotonically decreasing TC due to anharmonic phonon-phonon scattering. 
These clear failures ${ }^{29-32}$ of the current theory have prompted our rethinking of phonon transport in general, with a random alloy used here as an example.

\section{A revised perspective}

The fundamental problem with applying the PGM/VCA to an alloy is the presumption that all of the phonons/modes correspond to plane waves thereby justifying invocation of expressions for TC that are based on the PGM. In reality, when one adds a dissimilar atom or a defect/impurity into a previously pure homogenous crystal, one breaks the symmetry/periodicity that gave rise to all plane wave like solutions to the equations of motion. As a result, one obtains solutions to the equations of motion for the atoms that have a very different character than a plane wave modulated distribution of atom displacement/velocities (see Fig. 1b, c). Here, we used the eigenvector periodicity (EP) approach ${ }^{33}$ recently developed by Seyf and Henry to analyze the modes in a random alloy, namely $\ln _{1-x} \mathrm{Ga}_{x}$ As. Interestingly, as predicted by Allen and Feldman, the eigenvectors (e.g., the displacement/velocity fields ${ }^{33}$ ) for the normal modes in a crystalline random alloy fall into the same three categories identified by Allen and Feldman in 1999 for amorphous materials, namely propagons, diffusons and locons. ${ }^{10}$

Propagons are delocalized modes with sinusoidally modulated velocity field that exhibit a rather identifiable wavelength and correspond to low frequencies that in concept must occur in the low frequency limit as one recovers sound waves. Diffusons are delocalized modes (i.e., they extend through the entire system) that do not exhibit periodicity or a sinusoidally modulated velocity field, but instead appear to exhibit random vibrations similar to the randomized structure/composition itself. Lastly, locons correspond to localized vibrations that often center on atoms with significant deviations in local coordination than the rest of the structure. Localized modes can be distinguished from propagons and diffusons using the participation ratio ${ }^{34}$ defined as,

$$
\mathrm{PR}_{n}=\frac{\left(\sum_{i} \vec{e}_{i, n}{ }^{2}\right)^{2}}{N \sum_{i} \vec{e}_{i, n}{ }^{4}},
$$

where $\vec{e}_{i, n}$ is the eigenvector, $N$ is the number of atoms in the system, $n$ is the mode index, and index $i$ runs over all the atoms in the supercell. The above definition implies that the extended modes have a large value of $\mathrm{PR}_{n}$ whereas localized modes have small ratios that can reach a minimum value of $1 / \mathrm{N}$ for a mode completely localized on a single atom. In concept, locons are modes that involve a small minority of the system and typically have $\mathrm{PR}_{n}$ values below 0.15 . However, it is important to point out that the spectrum of PR values is continuous and there is in general no strict set of rules that would require any abrupt shift in mode character, as has been shown by Seyf and Henry. ${ }^{33}$

An important challenge that remained after the seminal work by Allen and Feldman was the issue of how to rigorously distinguish between the two types of delocalized modes, namely propagons and diffusons. This issue, however, was overcome by Seyf and Henry, who introduced the EP approach, which classifies modes on an individual and universal basis by purely considering their individual mode character, as opposed to a frequency or loffe-Rogel cross-over-based argument, which relies on an assessment of many modes collectively. This EP method uses the equilibrium atomic positions and eigenvectors of atoms in given vibrational mode and then calculates the degree of periodicity in the mode's velocity field. It then compares the EP of a mode to another fictitious mode that has pure sinusoidal modulation. In this way, the method normalizes the EP so that every mode falls between zero and unity. The extremes of zero and unity then correspond to a 0 and $100 \%$ sinusoidal/ propagating velocity field for a given mode. A detailed derivation of the EP, which measures the degree of spatial periodicity for the eigenvectors, has been given by Seyf and Henry, ${ }^{33}$ but here we briefly explain the approach. The degree of periodicity of eigenvectors in a mode can be calculated by

$$
\Psi(\overrightarrow{\mathbf{k}}, \varphi)=\left|\sum_{i} \sum_{j \geq i}\left[\vec{e}_{i, n} \cdot \vec{e}_{j, n}\right]\left[f\left(\overrightarrow{\mathbf{k}} \cdot \overrightarrow{\mathbf{r}}_{\mathbf{i}}+\varphi\right) f\left(\overrightarrow{\mathbf{k}} \cdot \overrightarrow{\mathbf{r}}_{j}+\varphi\right)\right]\right|,
$$

where $\|\overrightarrow{\mathbf{k}}\|=2 \pi / \lambda$ and $\varphi$ are the wave vector and phase of a periodic wave propagating in the structure, respectively. Variables $\overrightarrow{\mathbf{r}}$ and $\vec{e}$ represent the atom position vector and eigenvector, respectively. The function $f$ represents the periodic function chosen for comparison. Here, any spatially oscillatory function such as $\sin (\overrightarrow{\mathbf{k}} \cdot \overrightarrow{\mathbf{r}}), \cos (\overrightarrow{\mathbf{k}} \cdot \overrightarrow{\mathbf{r}})$, or $\exp (i \cdot \overrightarrow{\mathbf{k}} \cdot \overrightarrow{\mathbf{r}})$ can be used. The subscripts $i$ and $n$ refer to an atom and a mode in the structure, respectively. The function $\psi(\overrightarrow{\mathbf{k}}, \varphi)$ becomes large if the mode velocity field resembles that of the periodic function, and it provides a direct and quantitative measure of the degree of resemblance. Equation (2) can be normalized by comparing the value of $\psi(\overrightarrow{\mathbf{k}}, \varphi)$ for the actual mode, with $\psi(\overrightarrow{\mathbf{k}}, \varphi)$ for a fictitious mode that is based on the value of $\overrightarrow{\mathbf{k}}^{\prime}$ and $\varphi^{\prime}$ that maximize $\Psi(\overrightarrow{\mathbf{k}}, \varphi)$ for the mode in question. Thus, the appropriate fictitious mode for comparison is one that is oriented along the $\overrightarrow{\mathbf{k}}^{\prime}$ vector with phase $\varphi^{\prime}$. The final expression for normalized EP is then given by,

$$
\gamma_{n}=\frac{\left|\sum_{i} \sum_{j \geq i}\left[\vec{e}_{i, n} \cdot \vec{e}_{j, n}\right]\left[f\left(\overrightarrow{\mathbf{k}}^{\prime} \cdot \overrightarrow{\mathbf{r}}_{\mathbf{i}}+\varphi^{\prime}\right) f\left(\overrightarrow{\mathbf{k}}^{\prime} \cdot \overrightarrow{\mathbf{r}}_{j}+\varphi^{\prime}\right)\right]\right|}{\left|\sum_{i} \sum_{j \geq i}\left[\vec{s}_{i, n} \cdot \vec{s}_{j, n}\right]\left[f\left(\overrightarrow{\mathbf{k}}^{\prime} \cdot \overrightarrow{\mathbf{r}}_{\mathbf{i} 0}+\varphi^{\prime}\right) f\left(\overrightarrow{\mathbf{k}}^{\prime} \cdot \overrightarrow{\mathbf{r}}_{j 0}+\varphi^{\prime}\right)\right]\right|},
$$

where $\vec{s}$ is the eigenvector of fictitious mode. The value of $\gamma_{n}$ therefore represents the degree of EP on a normalized scale from zero to unity. For a mode with $100 \%$ propagating character $\gamma_{n}=1$, while modes with $\gamma_{n}$ values far from unity correspond to nonpropagating modes and could be either localized or de-localized. It is important to note here that the demarcation between propagons and diffusons ( $E P \sim 0.2)$ was determined by direct inspection of the modes observed in amorphous materials and represents a rather universal transition regime that is applicable to any mode, regardless of what type of material it exists in (e.g., alloys, molecules defected crystals etc.). ${ }^{33}$ This demarcation of EP $=0.2$ was used herein to distinguish between propagons and diffusons in the $\ln _{1-x} \mathrm{Ga}_{x}$ As alloy and it is important to note that it was not used as a fitting parameter.

Figure 1 shows an example calculation of the fraction of modes that are propagons in $\operatorname{In}_{1-x} \mathrm{Ga}_{x} \mathrm{As}$ as a function of In content. Based on the data in Fig. 1, one might expect that an expression for alloy TC that is based on the PGM might exhibit significant errors in its description of TC vs. temperature for alloys in the $15-85 \%$ composition range, because they would be in a regime where less than $2 \%$ of the modes are propagons. Again, this is because the PGM is built on the assumption that all modes propagate, and thus when situations occur where this is not true, one would expect PGM-based theories/models to breakdown, e.g., for alloys in the 15-85\% composition range. Furthermore, there are clear qualitative resemblances between Fig. 1a-c that suggest that even in the dilute limit, the steep decrease in TC may be more so related to the change in mode character (see Supplementary Materials for example animations of modes with less than $100 \%$ plane wave character) as opposed to impurity scattering.

It is also remarkable that even in the dilute limit, for impurity concentrations between $0.1-2 \%$, most of the modes in what would generally be considered a rather pure crystal, are far from being pure plane waves. Figure $1 b, c$ shows examples of how the mode character evolves in this regime and it is clear that the eigenvectors retain the sinusoidal periodicity most strongly in 
the wave vector direction, but it is most quickly lost in the perpendicular directions. This realization is insightful, because it shows very clearly what aspects of a mode's propagating character are lost first and how the transition from propagon to diffuson occurs. Furthermore, given that the momentum of phonons ( $\hbar \mathbf{k})$ emanates from the assumption that phonons are plane waves, the results in Fig. 1b, c suggest that significant rethinking of how phonons in non-pure crystals interact with other quantum particles such as electrons, photons and neutrons is necessary. It should be emphasized here that the results in Fig. 1b, c suggest that "pure" for phonons implies impurity concentrations less than $0.1-1 \%$, yet many materials used in industrial applications are only $97-99 \%$ pure. The data in Fig. 1 also suggests that the reason the alloy TC drops quickly within the first $10 \%$ may be associated with the loss of propagating character as opposed to impurity scattering, which is another fundamental shift in thinking.

A key question then becomes, what are the respective contributions that each category of modes (e.g., propagons, diffusons and locons) makes to TC?-answering this question requires a different theory, because the existing PGM theory cannot be invoked for the diffusons and locons. This is because one cannot define their velocities. For propagons, one can find an associated wavelength ${ }^{10,33}$ and could still envision treating such modes via the PGM/VCA. However, propagons only comprise a small fraction of the modes for $15-85 \%$ compositions (see Fig. 1b) and thus, it is not clear a priori, that all other modes can simply be neglected. For diffusons and locons, one must use alternative methods for describing their potentially significant TC contributions. Towards this end, Lv and Henry ${ }^{4}$ have developed a very general approach termed Green-Kubo modal analysis (GKMA), which combines supercell lattice dynamics (SCLD) with the fully anharmonic dynamics generated by a molecular dynamics (MD) simulation to solve this problem. The GKMA approach involves a projection of the anharmonic atomic trajectories onto the normal mode shapes (e.g., the velocity fields ${ }^{33}$ ) for each mode, without making any assumptions or modifications to the formalism for the different mode types. In this sense the GKMA method can more directly assess a mode's TC contribution, without any invocation of the PGM, as one only needs to utilize the mode level contributions to each atom's velocity to then determine each mode's contribution to the heat flux operator in an equilibrium MD simulation. In this sense, the key attribute of the GKMA approach is that it describes phonon transport in terms of correlation, rather than scattering, which is a major shift in perspective from the current understanding. The GKMA approach has been discussed in detail in previous work ${ }^{4}$ but here we briefly explain the approach. First, the harmonic frequencies and eigenvectors are obtained from a SCLD calculation. To obtain the modal contributions to the velocity of each atom (e.g., $\dot{\mathbf{x}}_{i}(n, t)$ atom $i$, mode $n$ ) the atom velocities from MD are projected onto the normal mode basis. The detailed formulation is given in previous work by Lv and Henry, ${ }^{4}$ but in the end the heat flux associated with each mode is calculated by substituting the modal velocity into heat flux operator,

$\mathbf{Q}(n, t)=\frac{1}{V} \sum_{i}\left[E_{i} \dot{\mathbf{x}}(n, t)+\sum_{j}\left(-\nabla_{\mathbf{r}_{\mathbf{i}}} \Phi_{j} \cdot \dot{\mathbf{x}}(n, t)\right) \mathbf{r}_{i j}\right]$,

where $E_{i}$ is the sum of potential and kinetic energy of atom $i, \Phi_{j}$ is the potential energy of atom $\mathrm{j}, V$ is the volume of the supercell, and $\mathbf{r}_{i j}$ is the distance between atom $j$ and atom $i$. Finally, the TC of each vibrational mode can be calculated by substituting the modal heat flux into the commonly used Green-Kubo expression,

$\kappa(n)=\frac{V}{k_{B} T^{2}} \int_{0}^{\infty}\langle\mathbf{Q}(n, t) \cdot \mathbf{Q}(0)\rangle d t$
Equation (5) expresses the TC as a direct summation over individual mode contributions. The reason it is useful to know the contributions of individual modes is multi-fold. For one, understanding the breakdown in mode level contributions allows each contribution to be associated with the mode's frequency. This is useful because one can then apply quantum heat capacity corrections, e.g., $\frac{x^{2} e^{x}}{\left(e^{x}-1\right)^{2}}$ to the TC contribution of every individual vibrational mode, where $x=\frac{h \omega}{k_{B} T}$, thereby allowing classical MD predictions to be extended to low temperatures where they are generally known to fail. ${ }^{6}$ The second advantage of knowing the mode level contributions is because one can then look at trends in the behaviors with respect to the mode character. For example, Gordiz and Henry $8,35,36$ observed profound features in the way different modes were correlated that had different characters. Similarly here, it would be useful to separate the contributions of propagons and diffusons to see if the trends in TC contributions or underlying mechanisms differ significantly, or if somehow despite the differences in character, the PGM physical picture still holds.

Thus far, the GKMA method has been tested and validated against various materials including, crystalline silicon, ${ }^{4}$ amorphous silicon, ${ }^{4}$ amorphous silica ${ }^{8,37}$ and amorphous carbon, ${ }^{6}$ yielding excellent agreement with experiments in every case, via a single unified formalism. Given the GKMA method's generality and accuracy, in the following analysis we have used it to study an example random alloy, namely $\ln _{1-x} G a_{x} A s$, and we compare its results to that of the PGM/VCA in order to test the hypothesis that the key fundamental information missing in the VCA is knowledge of the mode character.

\section{RESULTS AND DISCUSSION}

To illustrate why considering the mode character matters, we first modeled the $\ln _{1-x} \mathrm{Ga}_{x}$ As system using both VCA and GKMA. We then determined the TC as a function of composition at room temperature and then separately studied the TC of the $\ln _{0.53} G_{0.47}$ As alloy vs. temperature for several film thicknesses. The accuracy of the interatomic potential was verified first by its predictions of the TC vs. temperature for the IPHCs (InAs and $\mathrm{GaAs}$ ) separately. These calculations show that in the limit of an IPHC, the PGM and GKMA are consistent. Good agreement with experiments is also obtained for the TC vs. composition for $\mathrm{In}_{1-}$ ${ }_{x} \mathrm{Ga}_{x} \mathrm{As}$ (see Fig. 1a) at $300 \mathrm{~K}$, when using both methods. However, some differences between the two approaches show up in the predictions of the TC vs. temperature for the alloy.

One reason temperature reveals some discrepancy between the two methods is because phonon contributions to TC are proportional to the individual phonon heat capacities. Quantum mechanically, the heat capacity of phonons is strongly temperature dependent and decays to zero in a temperature range determined by the mode's frequency. At low temperatures, only low-frequency modes are excited, due to Bose-Einstein statistics, while the high-frequency modes only become active at higher temperatures. As a result, temperature serves as a rather broadband filter for the mode-level contributions to TC. Consequently, at low temperatures, one can single out the contributions each model assigns to low frequency modes, while at higher temperatures, higher frequency mode contributions are also included. Thus, one can probe the non-specific heat-related TC contributions for each method by comparing TC vs. temperature, because the specific heat portion of each respective model is identical-leaving the major distinction as each model's description of the phonon-phonon interactions. Figure 2 shows the TC calculated by each methodology as compared to the experimental data $^{38,39}$ for a thick 1.6 micron film, which are likely the bulk values. The results show that the VCA values are in the correct range, but the trends with temperature differ somewhat from the experimental data. 


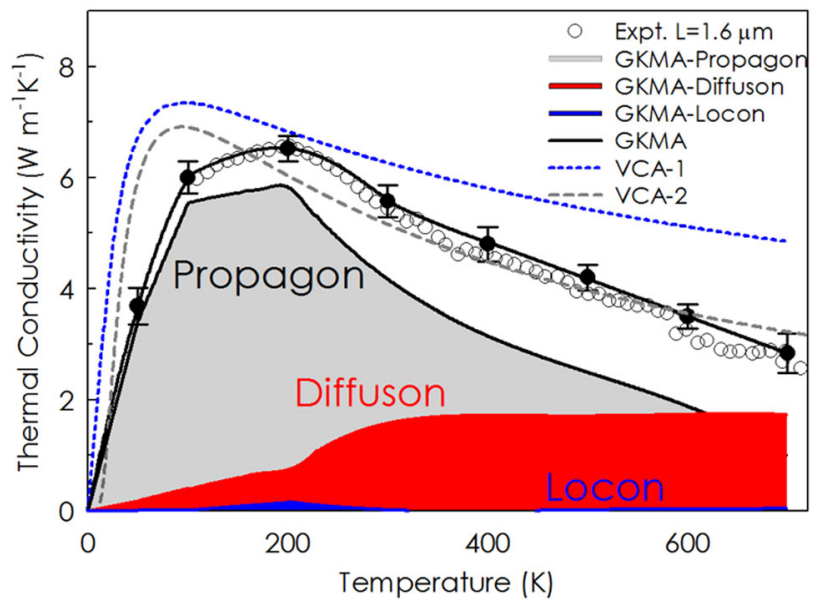

Fig. 2 TC of $\ln _{0.53} G_{0.47}$ As. Temperature dependent TC of $\ln _{0.53} \mathrm{Ga}_{0.47}$ As film ${ }^{38}, 39$ and the corresponding theoretical predictions using VCA and GKMA. The error bars were determined based on the standard deviation of GK results at a given temperature. Each labeled curve highlights the respective contributions associated with propagons, diffusons and locons, according to the GKMA and EP methodologies

Although there are some appreciable differences between the two methodologies, the VCA predictions of total TC are not drastically different from the experimental data, which might then lead one to assume that, maybe for this specific system at least, the physical picture described by the VCA is still valid. Furthermore, it might seem as though considering the mode character shift illustrated in Fig. 1b, c, is unnecessary. However, if this is true, the VCA should still provide correct guiding intuition and predictions for cases beyond the bulk crystalline behavior, and we are specifically interested in investigating a case that will highlight the difference between the two physical pictures (i.e., PGM/VCA via scattering vs. GKMA via mode character and correlation). In this respect, we note that a fundamental difference between the modes of an IPHC (e.g., plane waves), which are similar to propagons, and the predominant modes (by number) in the alloy, namely diffusons, is that the diffusons cannot be associated with a well-defined velocity. This then rigorously prevents them from being associated with a corresponding mean free path (MFP), and because the addition of multiple diffuson velocity fields presumably does not yield a traveling wave packet, it is intuitive to expect that diffusons should not exhibit significant classical size effects. ${ }^{3}$ A classical size effect is a well-known phenomenon that is well explained by the PGM and is one of the most valuable pieces of intuition it provides. ${ }^{3}$ According to the PGM, TC is proportional to the average distance a phonon can travel before it scatters (i.e., its MFP). Therefore, as one shrinks the size of a material, the propagation of phonons becomes constrained by the material's boundaries where it must scatter/ reflect. As a result, the reduced size of a material eventually limits the MFPs and consequently reduces TC in a predictable way. ${ }^{1,3,5}$ Diffusons and locons, however, do not propagate, and thus it is intuitive that they should not experience such size effects. Therefore it is intuitive that their contributions to TC should be unaffected by reduced dimensions, i.e., in a thinner film. It is important to also highlight here that locons have an associated length scale which can be quantified by a localization length, ${ }^{10}$ but diffusons do not since they are delocalized. Although Allen and Feldman ${ }^{10}$ proposed a length scale $\left(I^{2} \omega\right)$ associated with the mode diffusivity $\left(D_{i}(\omega)\right)$, to our knowledge this definition has never been shown to be a useful quantity.

Nonetheless, from this progression of logic, we compared the predictions of both methods (GKMA and VCA) to the TCs of much

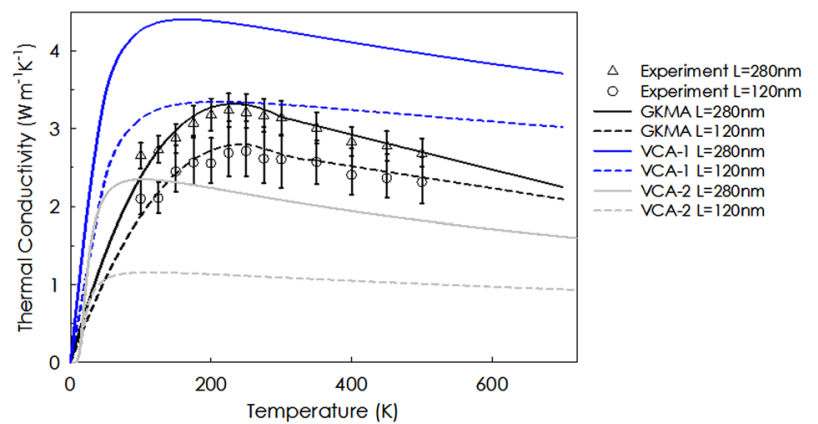

Fig. $3 \mathrm{TC}$ of $\mathrm{In}_{0.53} \mathrm{Ga}_{0.47}$ As thin films. Temperature-dependent TC of $\mathrm{In}_{0.53} \mathrm{Ga}_{0.47}$ As for different thin film thicknesses and the corresponding theoretical predictions using VCA and GKMA. Mathiessen's rule was used to apply boundary scattering to all the modes in VCA. For GKMA, the size effects were applied only to propagons by scaling their GKMA contributions by a reduction factor corresponding to the reduction in net relaxation time, which would occur after adding a boundary scattering term (diffuse limit) via Mathiessen's rule. In this approach, the base relaxation time for propagons was obtained via NMA. The error bars were determined based on the standard deviation of GK results at a given temperature

thinner films of $\ln _{0.53} \mathrm{Ga}_{0.47} \mathrm{As}$ (280 and $120 \mathrm{~nm}$ thick) whereby, according to the revised intuition, we hypothesized that the propagons would most certainly experience classical size effects, but the diffusons and locons should not. The details associated with the respective predictions and the subsequent experimental fabrication and measurements are described in detail in the Supplementary Materials. It should be emphasized here that the theoretical predictions were generated prior to the acquisition of the experimental data and thus served as a true test of the predictive capabilities of the respective methods/intuition. The VCA predictions were generated using the same methods used for the results shown in Fig. 2 and boundary scattering was applied to all the modes in the VCA using Mathissen's rule with the diffuse limit $L / 2 v$ boundary scattering relaxation time. ${ }^{1,3,17,20}$ It should be noted, that boundary scattering was also applied to the results in Fig. 2, however, because the film thickness was large (1.6 micron) it essentially had no effect on the TC results. Thus the only change between the predictions in Fig. 2 and Fig. 3 was the value of $L$, which was changed to the smaller film thicknesses for the results in Fig. 3. Based on our revised understanding for GKMA, size effects were applied to the propagons only, and not the diffusons or locons. The propagon relaxation times were calculated using the standard normal mode analysis (NMA) technique pioneered by McGaughey and Kaviany ${ }^{40}$ and the propagon contributions determined from GKMA were then scaled down according to the decreased relaxation times the propagons would experience in the thinner films, i.e.,

$\kappa_{\text {propagons }}=\sum_{i \geq E P P_{\text {cut }}}^{1} \kappa_{\mathrm{GKMA}} \frac{\tau_{i, \text { eff }}}{\tau_{i}}$

In the above equation $E P P_{\text {cut }}$ is the minimum eigenvector periodicity parameter of non-propagating modes, namely 0.2 . Above $\mathrm{EPP}=0.2$, vibrational modes behave like a plane wave vibrational mode and we assume their contributions to depend on MFPs as prescribed by the PGM. Here, $\tau_{i}$ is the net phonon-phonon and phonon-defect relaxation time calculated using NMA in MD and $\tau_{i, \text { eff }}$ is the effective net relaxation time after superimposing the effect of boundary scattering. This approach was used to avoid the excessively large computational expense associated with simulating the entire thickness of the film with free boundaries. Furthermore, the fact that an application of this basic intuition/ understanding can allow us to avoid the large computational expense associated with simulating the entire film, is precisely 
why it is so valuable to test and confirm that the intuition is correct in the first place. The predictions were then compared to TC measurements determined by time domain thermoreflectance measurements of single crystalline $\ln _{0.53} \mathrm{Ga}_{0.47}$ As thin film samples that were grown on single crystalline $\ln P$ substrates via metal-organic chemical vapor deposition (see Supplementary Materials for details).

The results in Fig. 3 show that the GKMA approach exhibits remarkable agreement with the experimental data and most notably, it properly captures the correct trends and magnitudes for each film. The VCA, on the other hand, provides a much poorer description as the added phenomenon of size effects exacerbates its key shortcoming. Of particular concern is the fact that both applications of the VCA (e.g., VCA-1 and VCA-2) incorrectly predict a lower-temperature peak $\sim 50-100 \mathrm{~K}$, while only the GKMA method correctly predicts that the peak occurs slightly above $200 \mathrm{~K}$.

It is also interesting to note that even if one discards the arguably more rigorous implementation of the VCA (e.g., VCA-1) in favor of the approach utilized more frequently before the advent of first principles methods (VCA-2), namely using fitting parameters, one still cannot properly predict the size effects seen in the thin films. When fitting parameters are used, it is interesting that the thicker 1.6 micron film TC and the overall shape of the TC accumulations (see Supplementary Materials) more closely match that predicted by the GKMA over the entire temperature range. However, because the PGM/VCA implicitly assumes that all of the modes are plane wave like in nature and should therefore experience size effects, the fitted version significantly underpredicts the TC of the films (i.e., error $>2 X$ for the $120 \mathrm{~nm}$ at $300 \mathrm{~K}$ ). Nonetheless, by more properly accounting for the fact that the majority of the modes are diffusons, which should not experience significant size effects, the GKMA predictions agree well with the measurements.

In conclusion, the results show that in a random alloy, the mode character changes dramatically with composition and the steep drop in TC between $0-2 \%$ corresponds with a decrease in plane wave/propagating character. This provides an alternative explanation to the more widely held understanding that associates this drop with impurity scattering. Furthermore, when $15-85 \%$ of the lattice sites are occupied by dissimilar atoms, the diffusons become the predominant mode type (by number-see Fig. 1b, c) and at high temperatures they can dominate the TC. The results also show that, consistent with their character, diffusons do not experience significant size effects at the $100 \mathrm{~nm}$-length scales probed herein, and as a result the TC of thin films differs significantly from VCA predictions, even when fitting parameters are employed. Thus, the theory of alloy TC and the concept of phonons more generally should be reconsidered to account for changes in mode character vs. disorder. Such rethinking has far reaching implications for phonon interactions with other quantum particles such as neutrons, electrons and photons, since the momentum associated with diffusons and locons is unclear. ${ }^{41-49}$ Furthermore, the results herein have shown that phonons cannot in general be thought of exclusively as plane waves. Instead, we believe a more general perspective is to classify phonons according to the three groups identified by Allen and Feldman, ${ }^{10}$ and more generally think of a phonon as "a quanta of normal mode vibrational energy". Ultimately the value and importance of these classifications is that they account for the strong possibility that each classification may have its own distinct physics for how it transports heat and interacts with other quantum particles (e.g., electrons, photons, neutrons etc.).

\section{METHODS}

The equilibrium MD simulations were performed using the large-scale atomic/molecular massively parallel simulator (LAMMPS). Prior to collecting statistics, structural relaxation was performed using a constant temperature, number of atoms, and volume for $500 \mathrm{ps}$. All simulations were run with a $0.5 \mathrm{fs}$ time step under the microcanonical ensemble for $15 \mathrm{~ns}$ to collect sufficient statistics for the TC calculations. One way to reduce computational expense in application of the GKMA method is to skip time steps when computing the modal contributions. The effect of the size of the computational domain on TC was also studied carefully, and we observed that a $18 \times 18 \times 18$ supercell was sufficiently large to include converged contributions of long wavelength phonons (e.g., propagons). We have calculated the modal TC contributions at eight temperatures for the $\operatorname{In}_{0.53} \mathrm{Ga}_{0.47} \mathrm{As}$ system. For the intermediate temperatures, we linearly interpolated the TC of each mode, using data for 50, 100, 200, 300, 400, 500,600 , and $700 \mathrm{~K}$. The GKMA results of individual mode TC are then multiplied by the quantum-corrected specific heat at the temperature of interest,

$\kappa_{T}(n)=\frac{\kappa_{T_{1}}(n)\left(T_{2}-T\right)+\kappa_{T_{2}}(n)\left(T-T_{1}\right)}{\left(T_{2}-T_{1}\right)}$.

To calculate each vibrational mode's relaxation time, in order to apply the effect of boundary scattering to the propagons, the atomic trajectories generated by MD simulations were also used. The normal modes amplitudes $S(\mathbf{k}, \boldsymbol{u})$ can be expressed as a sum over the positions of the atoms in the system.

$S_{i}(\mathbf{k}, u)=N^{-1 / 2} \sum_{j} M_{j}^{1 / 2} \exp \left(-i \mathbf{k} \cdot \mathbf{r}_{j, o}\right) \mathbf{e}_{i}^{*}(\mathbf{k}, u) \cdot\left(\mathbf{r}_{j}(t)-\mathbf{r}_{j 0}\right)$,

where $\mathbf{e}_{i}(\boldsymbol{k}, u)$ is the eigenvector, $u$ is mode polarization, $M_{j}$ is mass of atom $\mathrm{j}, \mathbf{k}$ is wave vector, $N$ is total number of atoms in the system, $\mathbf{r}_{j 0}$ and $\mathbf{r}_{j}(t)$ are the equilibrium lattice position and the atom's position vectors, respectively. by

Under harmonic approximation, the total energy of each mode is given

$E_{i}(t)=E_{j, P}+E_{j, K}=\frac{\omega_{i}^{2} S_{i} S_{i}^{*}}{2}+\frac{\dot{S}_{i} \dot{S}_{i}^{*}}{2}$,

where ${ }^{*}$ denotes complex conjugate and $\omega_{i}$ is the frequency of a given normal mode. The first term in Eq. (9) corresponds to the potential energy $\left(E_{j, P}\right)$ and the second term corresponds to the kinetic energy $\left(E_{j, k}\right)$. The vibrational mode relaxation time can be calculated from the decay of the autocorrelation of each mode's total energy via,

$\tau_{i}=\frac{\int_{0}^{\infty}\left\langle E_{i}(t) E_{i}(0)\right\rangle d t}{\left\langle E_{i}^{2}(0)\right\rangle}$

The eigenvectors are calculated using General Utility Lattice Program (GULP) at the gamma point $(\mathbf{k}=0)$, which represents a SCLD calculation, whereby the entire system is treated as the basis of a simple cubic crystal, as opposed to a more standard Lattice Dynamics calculation that exploits symmetry within the supercell. It is important to note that the relaxation times calculated using this method measure the net phonon-phonon scattering rate and it doesn't distinguish between umklapp and normal processes or the interactions with the alloying elements. This is due to the comprehensive inclusion of temperature dependent anharmonicity expressed through the atomic trajectory.

To calculate the TC using VCA, we first calculated harmonic and anharmonic interatomic force constants (IFCs) using the direct displacement method ${ }^{17,50}$ and then by using Fermi's golden rule, the anharmonic phonon lifetimes were calculated. ${ }^{14,15}$ The total lattice TC was determined under the relaxation time approximation by summing up the modal contributions. The ground state energy of a crystal can be expressed in terms of IFCs by Taylor expanding about the equilibrium positions,

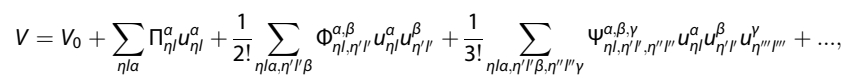

where $\Phi$ and $\psi$ are harmonic and cubic IFCs, respectively. Subscripts $a, \beta$, and $\gamma$ indicate the direction of the Cartesian displacement $u$ from the equilibrium position. Indices denote the $\eta^{\text {th }}$ atom in $t^{\text {th }}$ primitive cell. The residual force $\Pi$ is zero since the potential is expanded around the minimum energy configuration, hence,

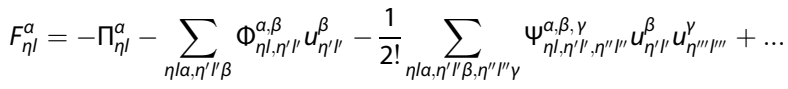


The IFCs are obtained by the real-space direct displacement method. ${ }^{17,50}$ In this approach, various sets of force-displacement data are calculated by displacing atoms in the supercell. Then, the displacement-force data are fitted to Eq. (9), taking the translational and rotational invariances into account. In the present study, we have considered only up to the cubic terms. For further details, readers are directed to the associated references. ${ }^{17,50}$

To model the atomic interactions and calculate the forces we used an empirical potential, namely the Abell-Tersoff potential, which was optimized using $a b$ initio data. ${ }^{51}$ The potential is able to correctly reproduce DFT-LDA calculated values of the elastic properties, cohesive energy, lattice constants and nonlinear effects in the strain within $1.2 \%{ }^{51}$ Before sampling force-displacement data, the cell parameter was optimized and we have obtained lattice parameter of $6.058 \AA$ for $\ln A s$ and $5.653 \AA$ for GaAs, which is in excellent agreement with the experimental values ${ }^{52}$ of $6.058 \AA$ and $5.653 \AA$, respectively. We then used a cubic supercell of a $2 \times 2 \times 2$ conventional unit cell, which consists of 64 atoms in total, to compute the various terms in Eq. (9).

After obtaining harmonic IFCs, the dynamical matrix for a given wave vector $\boldsymbol{k}$ can be calculated by Fourier transformation,

$D_{\eta \eta^{\prime}}^{\alpha, \beta}(\mathbf{k})=\frac{1}{\sqrt{M_{\eta} M_{\eta^{\prime}}}} \sum_{l^{\prime}} \Phi_{\eta 0, \eta^{\prime \prime} l^{\prime}}^{\alpha, \beta} e^{i q \cdot \mathbf{R}^{\prime}}$,

where $\mathbf{R}$ and $M$ are atomic position of the primitive cell and mass, respectively. The cubic IFCs are used to scattering matrix elements given by

$$
\begin{aligned}
& V_{3}\left(\mathbf{k} s, \mathbf{k}^{\prime} s^{\prime}, \mathbf{k}^{\prime \prime} s^{\prime \prime}\right)=\left(\frac{h}{8 N_{0} \omega(\mathbf{k} s) \omega\left(\mathbf{k}^{\prime} s^{\prime}\right) \omega\left(\mathbf{k}^{\prime \prime} s^{\prime \prime}\right)}\right)^{1 / 2} \\
& \sum_{\eta} \sum_{\eta^{\prime \prime \prime}} \sum_{\eta^{\prime \prime \prime} l^{\prime \prime}} \sum_{\alpha \beta \gamma} \psi_{0 l, \eta^{\prime \prime}, \eta^{\prime \prime} l^{\prime \prime}}^{\alpha, \beta,} e^{i \mathbf{k}^{\prime} \cdot \mathbf{R}^{\prime},} e^{i \mathbf{k}^{\prime \prime} \cdot \mathbf{R}^{\prime \prime}} \frac{e_{\eta}^{\alpha}(\mathbf{k} s) e_{\eta^{\prime}}^{\beta}\left(\mathbf{k}^{\prime} s^{\prime}\right) e_{\eta^{\prime \prime}}^{\gamma}\left(\mathbf{k}^{\prime \prime} s^{\prime \prime}\right)}{\sqrt{M_{\eta} M_{\eta^{\prime}} M_{\eta^{\prime \prime}}}}
\end{aligned}
$$

Where $N_{0}$ is the total number of mode in the first Brillouin zone, $\hbar$ is the Planck constant divided by $2 \pi$, and $s$ denotes different polarizations. Phonon lifetimes due to umklapp and normal three-phonon scattering processes can then be calculated using Fermi's golden rule, ${ }^{14,15}$

$$
\begin{aligned}
\frac{1}{\tau_{\mathbf{k s}}}= & \pi \sum_{\mathbf{k}^{\prime} s^{\prime}} \sum_{s}\left|V_{3}\left(\mathbf{k} s, \mathbf{k}^{\prime} s^{\prime}, \mathbf{k}^{\prime \prime} s^{\prime \prime}\right)\right|^{2} \\
& \times\left[2\left(n_{\mathbf{k}^{\prime} s^{\prime}}-n_{\mathbf{k}^{\prime \prime} s^{\prime \prime}}\right) \delta\left(\omega(\mathbf{k} s)+\omega\left(\mathbf{k}^{\prime} s^{\prime}\right)-\omega\left(\mathbf{k}^{\prime \prime} s^{\prime \prime}\right)\right)\right. \\
& \left.+\left(1+n_{\mathbf{k}^{\prime} s^{\prime}}+n_{\mathbf{k}^{\prime \prime} s^{\prime \prime}}\right) \delta\left(\omega(\mathbf{k} s)-\omega\left(\mathbf{k}^{\prime} s^{\prime}\right)-\omega\left(\mathbf{k}^{\prime \prime} s^{\prime \prime}\right)\right)\right]
\end{aligned}
$$

where $n_{\mathbf{k}}$ is the Bose-Einstein distribution. Conservation of momentum requires $\mathbf{k}+\boldsymbol{k}^{\prime}+\boldsymbol{k}^{\prime \prime}=\mathbf{G}$, where $\mathbf{G}$ is the reciprocal lattice vector. For normal process $\boldsymbol{G}=0$, while for Umklapp process $\boldsymbol{G} \neq 0$. Finally $\mathrm{TC}$ can be calculated based on relaxation time approximation,

$\kappa=\frac{1}{3 \Omega N_{0}} \sum_{\mathbf{k} s} v_{\mathbf{k s}}^{2} \tau_{\mathbf{k} s} \hbar \omega_{\mathbf{k} s} \frac{\partial n_{\mathbf{k} s}}{\partial T}$,

where $v_{\mathbf{k s}}$ is the group velocity and $\Omega$ is the volume of unit cell. We used a $30 \times 30 \times 30$ k-mesh within the first Brillouin zone, and we have confirmed that the TC is converged with respect to grid size when a $30 \times 30 \times 30$ grid is used.

The VCA introduced by Abeles ${ }^{13}$ was used to take into account the alloying effects. In this approach the disordered crystal is replaced with an ordered one with compositionally weighted IFCs, atomic mass and lattice constant according to composition. ${ }^{22}$ The mass disorder and anharmonicity are both treated as a perturbation. The net scattering rate of a phonon mode is calculated as the sum of scattering rate due to mass disorder and anharmonicity, according to Matthiessen's rule:

$\frac{1}{\tau_{\mathbf{k} s}}=\frac{1}{\tau_{\mathbf{k} s}^{p-p}}+\frac{1}{\tau_{\mathbf{k} s}^{m}}$

The first term is the phonon-phonon scattering rate which is calculated in the same way as the IPHCS except that for the virtual crystal, the compositionally weighted mass and lattice constants are used. The second term is the Tamura harmonic mass disorder scattering rate, which is calculated by using perturbation theory: ${ }^{11,14,15,22-25,27,28}$

$\frac{1}{\tau_{\mathbf{k} s}^{m}}=\frac{\pi}{2 N} \omega_{\mathbf{k} s}^{2} \sum_{\mathbf{k}^{\prime} s^{\prime}} \delta\left(\omega_{\mathbf{k} s}-\omega_{\mathbf{k}^{\prime} s^{\prime}}\right) \sum_{\sigma} g_{2}(\sigma)\left|\mathbf{e}_{\mathbf{k}^{\prime} s^{\prime}}^{*}(\sigma) \cdot \mathbf{e}_{\mathbf{k} s}(\sigma)\right|^{2}$, where $g_{2}(\sigma)=\sum_{i} f_{i}(\sigma)\left[1-m_{i}(\sigma) / m_{i e f f}(\sigma)\right]^{2}, f_{i}(\sigma)$ and $m_{i}(\sigma)$ are the atomic concentration and mass of ith isotope of the $\sigma$ atom. The quantity $m_{i, \text { eff }}(\sigma)=\sum_{i} f_{i}(\sigma) m_{i}(\sigma)$, is the weighted average mass of atom $\sigma$ and $\mathbf{e}$ is its polarization vector. This method has been applied to calculate TC of disordered alloys, such as Si-Ge alloy, ${ }^{22} \mathrm{PbTe}_{(1-x)} \mathrm{Se}_{x^{\prime}}{ }^{24}\left(\mathrm{Bi}_{(1-x)} \mathrm{Sb}_{x}\right)_{2} \mathrm{Te}_{3},{ }^{25}$ and $\mathrm{Mg}_{2} \mathrm{Si}_{x} \mathrm{Sn}_{1-x}{ }^{28}$ previously.

Finally the size effects are introduced via Matthiessen's rule,

$\frac{1}{\tau_{\mathbf{k} s, \mathrm{eff}}}=\frac{1}{\tau_{\mathbf{k} s}}+\frac{1}{\tau_{\mathbf{k s}, b}}$

where the second term is the boundary scattering rate, $\tau_{k s k}^{-1}=L / 2||_{\mathbf{k s}} \mid$

Using this approach, the room temperature bulk TC of $\ln _{0.53} \mathrm{Ga}_{0.47} \mathrm{As}$ using first-principles and the VCA by Vermeersc et al..$^{53}$ is calculated as $8.28 \mathrm{~W} \mathrm{~m}^{-1} \mathrm{~K}^{-1}$ while the prediction using the same methodology, but where the atomic interactions are modeled by the EIP is $8.07 \mathrm{~W} \mathrm{~m}^{-1} \mathrm{~K}^{-1}$. This good agreement between the EIP and experiments further indicates that the EIP accurately describes the interatomic forces for the alloy sufficiently well for the purposes herein.

In this study, we also employed a second method for calculating the TC of the alloy using VCA, whereby fitting parameters were used to describe phonon-phonon and phonon-impurity relaxation times, via an expression originally developed by Wang and Mingo. ${ }^{54}$ In this approach the TC is given by,

$\kappa=\int_{0}^{\hbar \omega_{c / k_{B} T}} \frac{k_{\mathrm{B}}^{4} T^{3}}{2 \pi^{2} v \hbar^{3}} \tau(T, y) y^{4} \frac{e^{y}}{\left[e^{y}-1\right]^{2}} d y$,

where $k_{\mathrm{B}}$ is Boltzmann's constant, $T$ is temperature, $\hbar$ is Planck's constant divided by $2 \pi, y=\hbar \omega / k_{B T}$ is a dimensionless parameter, $\omega_{c}$ is the cut-off frequency and obtained using Debye model. The average velocity $v$ is calculated by $v=\left[(1-x) v_{\mathrm{GaAs}}^{-2}+x v_{\operatorname{lnAs}}^{-2}\right]^{-1 / 2}$, where $x$ is the InAs concentration and $v_{I n A s}$ and $v_{\mathrm{GaAs}}$ are the average speeds of sound in $\ln A s$ and $\mathrm{GaAs}$, respectively. The scattering time for a given frequency is related to individual processes via Mattheissen's rule

$\tau=\left(\tau_{\mathrm{p}-\mathrm{p}}^{-1}+\tau_{\mathrm{m}}^{-1}+\tau_{\mathrm{b}}^{-1}\right)^{-1}$,

where $\tau_{p-p^{\prime}}^{-1} \tau_{m}^{-1}$, and $\tau_{b}^{-1}$ are the umklapp, mass disorder, and boundary scattering times, respectively. These are given by

$\tau_{\mathrm{p}-\mathrm{p}}=\left[(1-x) \tau_{\mathrm{GaAs}}^{-1}+x \tau_{\operatorname{lnAs}}^{-1}\right]^{-1}, \tau_{\mathrm{m}}=\left[x(1-x) A \omega^{4}\right]^{-1}, \tau_{\mathrm{b}}^{-1}=L / 2 v$,

where,

$\tau_{\mathrm{GaAs}(\operatorname{InAs})}^{-1}=B_{\mathrm{GaAs}(\operatorname{InAs})} \omega^{2} \exp \left(-C_{\mathrm{GaAs}(\operatorname{InAs}) / T}\right)$,

where $L$ is film thickness and constants $A, B$, and $C$ are fitting parameters. For more detail about the model, the reader is directed to the associated reference. $^{54}$

Data availability

The data that support the findings of this study are available from the corresponding author upon request.

\section{ACKNOWLEDGEMENTS}

All molecular dynamics calculations were run on the Partnership for Advanced Computing Environment (PACE) computer cluster at the Georgia Institute of Technology. We acknowledge support from the National Science Foundation through a Career Award (1554050) for A. Henry.

\section{AUTHOR CONTRIBUTIONS}

H.R.S conducted all the simulations and prepared the thin film samples for TC measurements. T.D., M.J., J.K., and R.D fabricated the samples. S.G. and B.A.C. designed and supervised the experiments. L.Y. and T.L.B. performed the thermal conductivity measurements and the associated uncertainty analysis. W.L. contributed modeling insight, and all authors analyzed and discussed the data. H.R.S and A.H cOwrote the paper, and all authors commented on the manuscript. A.H. provided direction and supervised the study. 


\section{ADDITIONAL INFORMATION}

Supplementary information accompanies the paper on the npj Computational Materials website (https://doi.org/10.1038/s41524-017-0052-9).

Competing interests: The authors declare that they have no competing financial interests.

Publisher's note: Springer Nature remains neutral with regard to jurisdictional claims in published maps and institutional affiliations.

\section{REFERENCES}

1. Srivastava, G. P. The Physics of Phonons (CRC Press, New York, 1990).

2. Dove, M. T. Introduction to Lattice Dynamics (Cambridge University Press, New York, 1993).

3. Chen, G. Nanoscale Energy Transport and Conversion: a Parallel Treatment Of Electrons, Molecules, Phonons, And Photons (Oxford University Press, New York, 2005).

4. Lv, W. \& Henry, A. Direct calculation of modal contributions to thermal conductivity via Green-Kubo modal analysis. New J. Phys. 18, 013028 (2016).

5. Ziman, J. M. Electrons and Phonons (Oxford University Press, New York, 2001).

6. Lv, W. \& Henry, A. Phonon transport in amorphous carbon using Green-Kubo modal analysis. Appl. Phys. Lett. 108, 181905 (2016).

7. Wei, L. \& Asegun, H. Non-negligible contributions to thermal conductivity from localized modes in amorphous silicon dioxide. Sci. Rep. 6, 35720 (2016).

8. Gordiz, K. \& Henry, A. Phonon transport at interfaces: determining the correct modes of vibration. J. Appl. Phys. 119, 015101 (2016).

9. Lv, W. \& Henry, A. Examining the validity of the phonon gas model in amorphous materials. Sci. Rep. 6, 37675 (2016).

10. Allen, P. B., Feldman, J. L., Fabian, J. \& Wooten, F. Diffusons, locons and propagons: character of atomic vibrations in amorphous Si. Philos. Mag. B 79, 1715-1731 (1999).

11. Tian, Z., Lee, S. \& Chen, G. Heat transfer in thermoelectric materials and devices. J. Heat Transf. 135, 061605-061605 (2013).

12. Cahill, D. G. et al. Nanoscale thermal transport. II. 2003-2012. Appl. Phys. Rev. 1, 011305 (2014)

13. Abeles, B. Lattice thermal conductivity of disordered semiconductor alloys at high temperatures. Phys. Rev. 131, 1906-1911 (1963).

14. Esfarjani, K., Garg, J. \& Chen, G. Modeling heat conduction from first principles. Annu. Rev. Heat Transf. 17, 9-47 (2014).

15. Feng, T. \& Ruan, X. Prediction of spectral phonon mean free path and thermal conductivity with applications to thermoelectrics and thermal management: a review. J. Nanomater. 2014, 206370 (2014)

16. Lindsay, L., Broido, D. A. \& Reinecke, T. L. Ab initio thermal transport in compound semiconductors. Phys. Rev. B 87, 165201 (2013).

17. Tadano, T., Gohda, Y. \& Tsuneyuki, S. Anharmonic force constants extracted from first-principles molecular dynamics: applications to heat transfer simulations. J. Phys. Condens. Matter 26, 225402 (2014).

18. Tadano, T. \& Tsuneyuki, S. Self-consistent phonon calculations of lattice dynamical properties in cubic $\mathrm{SrTiO} 3$ with first-principles anharmonic force constants. Phys. Rev. B 92, 054301 (2015).

19. Jiawei, Z., Bolin, L. \& Gang, C. First-principles calculations of thermal, electrical, and thermoelectric transport properties of semiconductors. Semicond. Sci. Technol. 31, 043001 (2016).

20. Wang, X. \& Huang, B. Computational study of in-plane phonon transport in Si thin films. Sci. Rep. 4, 6399 (2014).

21. Cahill, D. G., Watson, S. K. \& Pohl, R. O. Lower limit to the thermal conductivity of disordered crystals. Phys. Rev. B 46, 6131-6140 (1992).

22. Garg, J., Bonini, N., Kozinsky, B. \& Marzari, N. Role of disorder and anharmonicity in the thermal conductivity of silicon-germanium alloys: a first-principles study. Phys. Rev. Lett. 106, 045901 (2011).

23. Shiomi, J., Esfarjani, K. \& Chen, G. Thermal conductivity of half-Heusler compounds from first-principles calculations. Phys. Rev. B 84, 104302 (2011).

24. Takuru, M., Takuma, S., Takuma, H., Keivan, E. \& Junichiro, S. Importance of local force fields on lattice thermal conductivity reduction in PbTe 1-x Se $\mathrm{x}$ alloys. Europhys. Lett. 102, 46002 (2013).

25. Katcho, N. A., Mingo, N. \& Broido, D. A. Lattice thermal conductivity of (Bi 1-x Sb x)2 Te3 alloys with embedded nanoparticles. Phys. Rev. B 85, 115208 (2012).

26. Tamura, S.-i Isotope scattering of dispersive phonons in Ge. Phys. Rev. B 27, 858-866 (1983)

27. Kundu, A., Mingo, N., Broido, D. A. \& Stewart, D. A. Role of light and heavy embedded nanoparticles on the thermal conductivity of SiGe alloys. Phys. Rev. B 84, 125426 (2011).

28. Li, W., Lindsay, L., Broido, D. A., Stewart, D. A. \& Mingo, N. Thermal conductivity of bulk and nanowire Mg2 Si $x$ Sn 1-x alloys from first principles. Phys. Rev. B 86, 174307 (2012).
29. Lee, S., Esfarjani, K., Mendoza, J., Dresselhaus, M. S. \& Chen, G. Lattice thermal conductivity of $\mathrm{Bi}, \mathrm{Sb}$, and $\mathrm{Bi}-\mathrm{Sb}$ alloy from first principles. Phys. Rev. B 89, 085206 (2014).

30. Liu, W. \& Balandin, A. A. Thermal conduction in AlxGa1-xN alloys and thin films. J. Appl. Phys. 97, 073710 (2005).

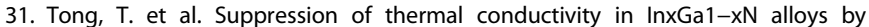
nanometer-scale disorder. Appl. Phys. Lett. 102, 121906 (2013).

32. Borca-Tasciuc, T. et al. Thermal conductivity of AlAs0.07Sb0.93 and Al0.9Ga0.1As0.07Sb0.93 alloys and (AIAs)1/(AISb) 11 digital-alloy superlattices. J. Appl. Phys. 92, 4994-4998 (2002).

33. Seyf, H. R. \& Henry, A. A method for distinguishing between propagons, diffusions, and locons. J. Appl. Phys. 120, 025101 (2016).

34. Biswas, R., Bouchard, A. M., Kamitakahara, W. A., Grest, G. S. \& Soukoulis, C. M. Vibrational localization in amorphous silicon. Phys. Rev. Lett. 60, 2280-2283 (1988).

35. Gordiz, K. \& Henry, A. Interface conductance modal analysis of lattice matched InGaAs/InP. Appl. Phys. Lett. 108, 181606 (2016).

36. Gordiz, K. \& Henry, A. A formalism for calculating the modal contributions to thermal interface conductance. New. J. Phys. 17, 103002 (2015).

37. Lv, W. \& Henry, A. Non-negligible contributions to thermal conductivity from localized modes in amorphous silicon dioxide. Sci. Rep. 6, 35720 (2016).

38. Kim, W. et al. Thermal conductivity reduction and thermoelectric figure of merit increase by embedding nanoparticles in crystalline semiconductors. Phys. Rev. Lett. 96, 045901 (2006).

39. Kim, W. et al. Cross-plane lattice and electronic thermal conductivities of ErAs: InGaAs/InGaAlAs superlattices. Appl. Phys. Lett. 88, 242107 (2006).

40. McGaughey, A. J. H. \& Kaviany, M. Quantitative validation of the Boltzmann transport equation phonon thermal conductivity model under the single-mode relaxation time approximation. Phys. Rev. B 69, 094303 (2004).

41. Balakrishnan, G., Bernhoeft, N. R., Bowden, Z. A., Paul, D. M. \& Taylor, A. D. Vibrational anomalies in the superconducting compound La1.85Ba0.15CuO4. Nature 327, 45-47 (1987).

42. Blencowe, M. Quantum physics: photons paired with phonons. Nature 530, 284-285 (2016)

43. Drozdov, A. P., Eremets, M. I., Troyan, I. A., Ksenofontov, V. \& Shylin, S. I. Conventional superconductivity at 203 kelvin at high pressures in the sulfur hydride system. Nature 525, 73-76 (2015).

44. Ernst, G., Broholm, C., Kowach, G. R. \& Ramirez, A. P. Phonon density of states and negative thermal expansion in ZrW2O8. Nature 396, 147-149 (1998).

45. Keppens, V. et al. Localized vibrational modes in metallic solids. Nature 395, 876-878 (1998)

46. Lanzara, A. et al. Evidence for ubiquitous strong electron-phonon coupling in high-temperature superconductors. Nature 412, 510-514 (2001).

47. LeRoy, B. J., Lemay, S. G., Kong, J. \& Dekker, C. Electrical generation and absorption of phonons in carbon nanotubes. Nature 432, 371-374 (2004).

48. Mook, H. A. \& Dogan, F. Charge fluctuations in YBa2Cu3O7-x high-temperature superconductors. Nature 401, 145-148 (1999).

49. Riedinger, R. et al. Non-classical correlations between single photons and phonons from a mechanical oscillator. Nature 530, 313-316 (2016).

50. Esfarjani, K. \& Stokes, H. T. Method to extract anharmonic force constants from first principles calculations. Phys. Rev. B 77, 144112 (2008).

51. Powell, D., Migliorato, M. A. \& Cullis, A. G. Optimized Tersoff potential parameters for tetrahedrally bonded III-V semiconductors. Phys. Rev. B 75, 115202 (2007).

52. Madelung, O. Semiconductors-Basic Data. (Springer, Berlin Heidelberg, 1996).

53. Vermeersch, B., Carrete, J. \& Mingo, N. Cross-plane heat conduction in thin films with ab-initio phonon dispersions and scattering rates. Appl. Phys. Lett. 108, 193104 (2016).

54. Wang, Z. \& Mingo, N. Diameter dependence of SiGe nanowire thermal conductivity. Appl. Phys. Lett. 97, 101903 (2010).

55. Maycock, P. D. Thermal conductivity of silicon, germanium, III-V compounds and III-V alloys. Solid State Electron. 10, 161-168 (1967).

Open Access This article is licensed under a Creative Commons Attribution 4.0 International License, which permits use, sharing, adaptation, distribution and reproduction in any medium or format, as long as you give appropriate credit to the original author(s) and the source, provide a link to the Creative Commons license, and indicate if changes were made. The images or other third party material in this article are included in the article's Creative Commons license, unless indicated otherwise in a credit line to the material. If material is not included in the article's Creative Commons license and your intended use is not permitted by statutory regulation or exceeds the permitted use, you will need to obtain permission directly from the copyright holder. To view a copy of this license, visit http://creativecommons. org/licenses/by/4.0/.

(c) The Author(s) 2017 